\title{
Antcin A contributs to anti-inflammatory effect of Niuchangchih (Antrodia camphorata)
}

\author{
Zhen-ming LU, Zheng-hong XU*
}

Acta Pharmacologica Sinica (2011) 32: 981-982; doi: 10.1038/aps.2011.104

$\mathrm{I}_{\mathrm{t}}^{\mathrm{n}}$ flammation is the responses of body to harmful stimuli in many pathological conditions. Prevention and treatment of inflammation are the main indication of a variety of natural products. Recently, Niuchangchih (Antrodia camphorata), a medicinal mushroom in Taiwan, has received considerable attention from the public due to its potent bioactivities, such as anti-inflammation, anti-cancer, immuno-modulatory and anti-hepatitis activities $^{[1]}$. Till now, researchers have identified a total of 26 compounds from Niuchangchih, including 11 succinic/ maleic acid derivatives, 8 triterpenoids, 1 benzenoids, 1 benzoquinone derivative, 5 miscellaneous compounds and polysaccharides, that possess antiinflammatory effect ${ }^{[2,3]}$. However, the mechanisms underlying their antiinflammatory actions remain elusive.

A recent study by Chen et al elucidated the molecular mechanisms of anti-inflammation of Niuchangchih. The authors isolated and purified 5 major antcins (A, B, C, H, and K) from fruiting bodies of Niuchangchih. They found that antcin A was most similar to glucocorticoids among the 5 compounds in the chemical structure. Furthermore, they

Laboratory of Pharmaceutical Engineering, School of Medicine and Pharmaceutics, Jiangnan University, Wuxi 214122, China Correspondence: Prof Zheng-hong XU (zhenghxu@jiangnan.edu.cn) demonstrated in human lung cancer cell A549 that antcin A was the active ingredient responsible for the anti-inflammatory effect of Niuchangchih, which might act via the same molecular mechanism triggered by glucocorticoids ${ }^{[4]}$. Finally, they showed the docking of antcin A to glucocorticoid receptor (GR) in a molecular modeling study. Thus, via mimicking glucocorticoids, antcin A may diffuse across the cell membrane and bind to the cytosolic GR that forms a dimmer after dissociated from the heat-shock protein (HSP) and then translocates into the nucleus to initiate the suppression of inflammation at the gene regulation level. Molecular docking showed that C-7 of antcin A was attached to the hydrophobic side of the steroidal backbone of GR, while C-7 of the other antcins was attached to the hydrophilic group, thus being expelled when docking to the binding cavity of GR.

Chen's work is an important landmark in research of anti-inflammatory compounds in Niuchangchih. Hsien et al recently showed that the anti-inflammatory activity of antrocamphin A, another anti-inflammatory compound from Niuchangchih, resulted from suppressing pro-inflammatory molecule release via down-regulation of iNOS and COX-2 expression through NF-KB pathway ${ }^{[5]}$. Both the studies provide solid evidence for the potential of Niuchangchih to treat inflammation. To elucidate the mechanisms of action of antcin A and antrocamphin A in vivo, more animal experiments and randomized controlled clinical trials should be carried out. Since more than 20 compounds in Niuchangchih have been shown to possess antiinflammatory activity ${ }^{[6-8]}$, further studies are needed to elucidate their mechanisms. It is also of interest to figure out whether these compounds act synergistically or independently. Notably, most anti-inflammatory compounds in Niuchangchih have been derived from the fruiting bodies so far. Further research is needed to uncover whether they can also be produced in fermented mycelia. Besides antcin A and antrocamphin A, many unknown ingredients responsible for the anti-infammatory effects of Niuchangchih remain to be discovered.

\section{References}

1 Ao ZH, Xu ZH, Lu ZM, Xu HY, Zhang XM, Dou WF. Niuchangchih (Antrodia camphorata) and its potential in treating liver diseases. J Ethnopharmacol 2009; 121: 194-212.

2 Geethangili M, Tzeng YM. Review of pharmacological effects of Antrodia camphorata and its bioactive compounds. Evid Based Complement Alternat Med 2011; 2011: 212641.

3 Chen CC, Liu YW, Ker YB, Wu YY, Lai EY, Chyau $\mathrm{CC}$, et al. Chemical characterization and antiinflammatory effect of polysaccharides fractionated from submerge-cultured Antrodia camphorata 
mycelia. J Agric Food Chem 2007; 55: 5007-12.

4 Chen YC, Liu YL, Li FY, Chang Cl, Wang SY, Lee $\mathrm{KY}$, et al. Antcin A, a steroid-like compound from Antrodia camphorata, exerts anti-inflammatory effect via mimicking glucocorticoids. Acta Pharmacol Sin 2011; 32: 904-11.

5 Hsien YH, Chu FH, Wang YS, Chien SC, Chang $\mathrm{ST}$, Shaw JF, et al. Antrocamphin A, an anti- inflammatory principal from the fruiting body of Taiwanofungus camphoratus, and its mechanisms. J Agric Food Chem 2010; 58: 3153-8.

6 Chen JJ, Lin WJ, Liao CH, Shieh PC. Anti-inflammatory benzenoids from Antrodia camphorata. J Nat Prod 2007; 70: 989-92.

7 Chien SC, Chen ML, Kuo HT, Tsai YC, Lin BF, Kuo $\mathrm{YH}$. Anti-inflammatory activities of new succinic and maleic derivatives from the fruiting body of Antrodia camphorata. J Agric Food Chem 2008; 56: 7017-22.

8 Wu SJ, Leu YL, Chen CH, Chao CH, Shen DY, Chan $\mathrm{HH}$, et al. Camphoratins A-J, potent cytotoxic and anti-inflammatory triterpenoids from the fruiting body of Taiwanofungus camphoratus. J Nat Prod 2010; 73: 1756-62. 